\title{
Bevacizumab: Zankapfel der Glioblastomtherapie
}

Hundsberger, Thomas ; Weller, Michael

DOI: https://doi.org/10.1024/1661-8157/a002650

Other titles: Bevacizumab: Bone of Contention in the Therapy of Glioblastoma

Posted at the Zurich Open Repository and Archive, University of Zurich ZORA URL: https://doi.org/10.5167/uzh-141049

Journal Article

Accepted Version

Originally published at:

Hundsberger, Thomas; Weller, Michael (2017). Bevacizumab: Zankapfel der Glioblastomtherapie. Praxis, 106(8):415-420.

DOI: https://doi.org/10.1024/1661-8157/a002650 
Bevacizumab: Zankapfel der Glioblastomtherapie

Bevacizumab: Bone of Contention in the Therapy of Glioblastoma

Thomas Hundsberger ${ }^{1,2}$, Michael Weller ${ }^{3}$

${ }^{1}$ Klinik für Neurologie, Kantonsspital, St. Gallen

${ }^{2}$ Klinik für Hämatologie und Onkologie; Kantonsspital, St. Gallen

${ }^{3}$ Klinik für Neurologie, Universitätsspital, Zürich

Korrespondenz:

PD Dr. Thomas Hundsberger

Klinik für Neurologie und

Klinik für Hämatologe und Onkologie

Kantonsspital

Rorschacherstrasse 95

9007 St. Gallen, Schweiz

Tel.: 0041714943095

Fax.: 0041714942895

Email: thomas.hundsberger@kssg.ch 
Abkürzungen

EGFR, epithelial growth factor receptor

GB, Glioblastom

Gd, Gadolinium

Iri, Irinotecan

Lom, Lomustin

MRT, Magnetresonanztomographie

mPFS, median PFS

mOS, median OS

PFS, progression free survival

PFS-6, progression free survival at 6 months

OS, overall survival

RANO, response assessment in neuro-oncology

Tem, Temozolomid

u.a., unter anderem

VEGF, vascular endothelial growth factor 
Zusammenfassung

Das Glioblastom rezidiviert regelmäßig trotz multimodaler Erstlinientherapie innerhalb weniger Monate nach der Diagnose. Für diese Situation ist bislang noch kein Therapiestandard etabliert. Bevacizumab, ein Antikörper gegen den vaskulären endothelialen Wachstumsfaktor, weckte große Hoffnungen in der Therapie des Glioblastoms. Zwei Phase III-Studien zeigten allerdings nur eine Verzögerung der Krankheitsprogression nicht aber des Gesamtüberlebens durch die Kombination der Standarderstlinientherapie mit Bevcizumab. Gleiches ergab die EORTC 26101-Rezidivtherapiestudie mit einer Kombination von Lomustin/Bevacizumab gegenüber einer Lomustin-Monotherapie. Zudem existieren widersprüchliche Beobachtungen zur Lebensqualität und Neurokognition. Trotz der insgesamt enttäuschenden Studienlage wird Bevacizumab aufgrund seines sehr guten palliativen Effektes von den Schweizer neuroonkologischen Zentren als zugelassene Therapieoption in der Rezidivtherapie häufig eingesetzt. Vakzinierungs- und Immuntherapien eröffnen neue Einsatzmöglichkeiten für Bevacizumab in der Behandlung primärer Hirntumore.

Schlüsselwörter

D: Glioblastom; vaskulärer endothelialer Wachstumsfaktor; Bevacizumab

E: Glioblastoma; vascular endothelial growth factor; bevacizumab 


\section{Ü1: Einleitung}

Das Glioblastom (GB) ist der häufigste und bösartigste hirneigene Tumor beim Erwachsenen. Histologisch ist er charakterisiert durch eine ausgeprägte Zellanaplasie, eine erhöhte Zellproliferation und das Nebeneinander von Gefäßproliferaten und von Nekrosen (WHO Definition). Wie bei den meisten bösartigen Tumoren beruht die Entstehung von Tumorzellen auf einem multifaktoriellen Prozess somatischer Mutationen. Der einzig gesicherte Risikofaktor für die Entstehung von GBs ist eine vorangegangene Bestrahlung des Gehirns [1]. Nur selten entsteht das GB auf dem Boden hereditärer Tumorsyndrome wie dem Li-Fraumeni Syndrom (Keimbahnmutation im p53 Gen) oder der Neurofibromatose Typ 1 (Keimbahnmutation im Neurofibromin-Gen) [2].

Das GB ist mit einer Inzidenz von 3,2/100000 Einwohner ein seltener Tumor. Für die Schweiz sind somit jährlich etwa 250 Neuerkrankungen zu erwarten [3]. Es ist eine Erkrankung des höheren Lebensalters (medianes Erkrankungsalter 64 Jahre) mit einer männlichen Prädominanz (1,6 Mal häufiger). Das Gesamtüberleben ist mit 37\% nach einem Jahr und nur $5 \%$ nach 5 Jahren sehr kurz [1]. Aufgrund dessen bedarf es erheblicher Anstrengungen und neuer therapeutischer Strategien, um diese Situation zu verbessern [4].

\section{Ü1: Pathophysiologie}

Das GB ist ein rasch proliferierender Tumor. Das Wachstum ist so dynamisch, dass die Blut-, Nährstoff- und Sauerstoffversorgung häufig nicht mehr Schritt halten kann. Aufgrund dessen entstehen hypoxische und konsekutiv nekrotische Zonen innerhalb des Tumors, die als histologisches Kardinalkriterium für die maligne Graduierung herangezogen werden. Die Hypoxie triggert über eine Ausschüttung von Hypoxie-induzierbaren Zytokinen unter anderem die Bildung des vaskulären endothelialen Wachstumsfaktors A (VEGF-A) [5]. Dieses Signal lässt neue Kapillaren aus bestehenden Blutgefäßen aussprossen. Darüber hinaus bilden auch die GB-Zellen VEGF und üben über einen parakrinen Mechanismus einen starken Proliferationsreiz auf Tumorgefäße aus. Diese sollen die Nährstoffversorgung und die Homöostase des Tumors sicherstellen. Allerdings sind diese rasch wachsenden Gefäße fragil und durchlässig. Regelmäßig treten Tumorhämorrhagien auf und der Austritt von intravaskulärer Flüssigkeit induziert ein charakteristisches, vasogenes Hirnödem. Ferner ist die Unreife der Tumorgefäße im Gegensatz zu der ansonsten gut abgedichteten Bluthirnschranke für den Austritt von Kontrastmittel als radiologisches Kennzeichen eines bösartigen, Gefäß-proliferativen Hirntumors verantwortlich. Dieses Hirnödem erhöht den intratumoralen Druck und reduziert die Tumorperfusion. 
Ü1: Der vaskuläre endotheliale Wachstumsfaktor Typ A

VEGF-A ist einer der am besten untersuchten und potentesten pro-angiogenetischen Wachstumsfaktoren [6]. Er ist in großen Mengen in peritumoraler Flüssigkeit und im Plasma von Patienten mit Glioblastomen nachweisbar [7]. Dabei ist die Überexpression von VEGF-A und das Ausmass der Gefässproliferation mit einer schlechten Prognose assoziiert [8, 9]. Neben der Neoangiogenese soll VEGF auch proliferationsstimulierende Effekte auf die Glioblastom(Stamm-)zellen selbst haben und als Wachstumsfaktor im zentralen Nervensystem dienen [9].

Die therapeutische Hemmung von VEGF optimiert die Tumorgefäßarchitektur und bewirkt eine transiente vaskuläre Normalisierung [10]. Neuere Arbeiten an Lungentumoren widersprechen jedoch dieser These [11]. Die Abnahme des intratumoralen Druckes und die verbesserte Tumorperfusion erleichtert zumindest theoretisch die Anflutung von Chemotherapeutika über die Blutbahn. Diese Wirkung wird durch anti-angiogenetische Substanzen wie den humanisierten, monoklonalen Anti-VEGF Antikörper Bevacizumab erreicht. Bevacizumab verhindert die Proliferation von Endothelzellen und somit die Neoangiogenese [5]. Attraktiv ist zudem der Wirkort der Substanz innerhalb des Gefäßlumens, so dass Bevacizumab als großes Molekül nicht die Bluthirnschranke überwinden muss, um seine Wirkung zu entfalten. Da eine Bestrahlung die Expression von VEGF triggern kann [12], bietet sich eine Kombination von Radiotherapie mit Bevacizumab an, um synergistische therapeutische Wirkungen in der Behandlung des GB zu entfalten. Diesem Konzept wurde in der AVAglio und der RTOG 0825 Studie Rechnung getragen $[13,14]$.

\section{Ü1: Studienlage zu Bevacizumab in der Behandlung des GB}

Die erste klinische Anwendung von Bevacizumab bei malignen Gliomen geht auf die Therapie von 21 Patienten mit rezidiviertem Tumor durch die Erfahrung einer niedergelassenen Onkologin zurück [15]. Sie kombinierte Bevacizumab mit Irinotecan in einer Dosierung, die für die Behandlung des kolorektalen Karzinoms etabliert worden ist und konnte ein Ansprechen bei 9 und eine Tumorstabilisierung bei 11 Patienten nachweisen. Die erfolgsversprechenden klinischen Ansprechraten konnten in der zwar randomisierten, jedoch nicht-komparativen BRAIN-Studie ( $n=167$ ) durch den Einsatz von Bevacizumab als Monotherapie (10 mg/kg; alle 14 Tage) und in der Kombination mit Irinotecan, einem Topoisomerase I-Inhibitor, beim rezidivierten GB mit einem progressionsfreien Überleben nach 6 Monaten (PFS-6) von 43\% bzw. 50\% reproduziert werden [16]. In 70\% der Patienten verbesserte sich der klinische Zustand und die Steroiddosis konnte reduziert werden. Warum die Bevacizumabdosis in dieser Studie verdoppelt wurde, bleibt unklar [17, 18]. Auf der Basis zweier unkontrollierter Phase-II Studien mit insgesamt 215 eingeschlossenen Patienten wurde Bevacizumab zwar in 
den USA und unter anderem in der Schweiz nicht jedoch durch die Europäische Arzneimittelbehörde zur Monotherapie des rezidivierten GB zugelassen [16, 17, 19]. Diese Zulassung auf dem Boden unzureichender, durch Studien abgesicherter Daten dokumentiert einerseits die hohe medizinische Notwendigkeit, verhinderte jedoch anderseits die Klärung zur Frage des optimalen Einsatzes dieser Substanz (u.a. Dosis, Zeitpunkt, Mono- versus Kombinationstherapie, Überlebensvorteil) $[17,18]$.

Im Jahr 2014 wurden die Ergebnisse der AVAglio- und der RTOG 0825-Studie zu Primärbehandlung des GBs mit multimodaler Standardtherapie +/- Bevacizumab publiziert [13, 14] (Tabelle 1). Beide Studien unterschieden sich in den Einschlusskriterien nur leicht (u.a. Resektion versus Biopsie/Resektion, Beginn und Dauer der Bevacizumabgabe, Messinstrumente der gesundheitsbezogenen Lebensqualität). Beide Studien wählten das mediane progressionsfreie (median progression free survival, mPFS) und das mediane Gesamtüberleben (median overall survival, mOS) als kombinierten primären Endpunkt. Leider enttäuschten die Ergebnisse beider Studien, da durch die Kombination der Standardtherapie mit Bevacizumab keine Verlängerung des mOS erreicht werden konnte (Tabelle 1). Allerdings verlängerte Bevacizumab das mPFS in beiden Studien in gleichem Ausmaß, jedoch aufgrund eines unterschiedlichen statistischen Designs nur signifikant in der AVAglio-Studie. Als eine der möglichen Ursachen für die fehlende Translation der Progressionsverlängerung in einen Überlebensvorteil wird der relevante Anteil an Patienten im Kontrollarm diskutiert, die bei Tumorprogression ebenfalls Bevacizumab erhalten haben (sogennantes crossover, AVAglio:31\%, RTOG 0825:48\%) [20].

In beiden Studien konnte die Steroiddosierung reduziert werden, jedoch stieg auch die Rate an relevanten Nebenwirkungen an. Hier sind insbesondere arterielle und venöse thromboembolische Ereignisse und die arterielle Hypertonie zu nennen, was für VEGFinhibitorische Substanzen typisch ist [21]. Nur in einer retrospektiven Analyse der AVAglioStudie konnte ein Überlebensvorteil in einer bestimmten molekularen Subgruppe (proneural, Isocitratdehydrogenese-Wildtyp GB) herausgearbeitet werden [22]. Diese Daten bedürfen jedoch der prospektiven Bestätigung. Im Gegensatz zur RTOG 0825-Studie, die eine Verschlechterung der Neurokognition, des Wortgedächtnisses und weiterer neuropsychologischer Test feststellte (sogenannter net clinical benefit), konnte die AVAglioStudie keine Verschlechterung aber auch keine Verbesserung der gesundheitsbezogenen Lebensqualität nachweisen [14, 23]. Die Ursache dieser kontroversen Ergebnisse ist weiterhin unklar.

Der Stellenwert von Bevacizumab in der Behandlung des rezidivierten GB wurde kürzlich ebenfalls in einer randomisierten Phase III-Studie untersucht (EORTC 26101) [24]. Ausgehend 
von den Daten einer dreiarmigen, prospektiven Phase II-Studie [25] war die Kombination von Bevacizumab und dem oralen Alkylanz Lomustin besser, als eine Monotherapie mit Lomustin alleine. Leider spiegelte sich das verlängerte mPFS der Kombinationstherapie (4,2 versus 1,5 Monate) wiederum nicht in einer Verbesserung des mOS wieder (8,6 versus 9,1 Monate).

Ü1: Wo liegt der Stellenwert von Bevacizumab zur Behandlung des GB im klinischen Alltag? Trotz der enttäuschenden Ergebnisse dreier Phase III-Studien bezüglich des OS ist Bevacizumab nach wie vor in der Schweiz zu Monotherapie des GB-Rezidivs zugelassen. Dies wird von vielen Schweizer neuro-onkologischen Zentren befürwortet, die diese Substanz als palliative Therapie insbesondere bei inoperablen und großen GB mit hohem Steroidbedarf regelmäßig einsetzen [26]. Aus der persönlichen Erfahrung ist Bevacizumab eine einfach zu handhabende Substanz mit wenigen, kalkulierbaren Nebenwirkungen, die durch eine gute Patientenschulung (Thromboembolien, gastrointestinale Perforation) und die regelmäßige Untersuchung des Urineiweiss und der Blutdruckwerte gut monitoriert werden können. Herausgestellt werden muss, das bei vielen Patienten mit einem Steroidbedarf aufgrund eines perifokalen Hirnödem die Cortisongabe entscheidend reduziert und häufig sogar abgesetzt werden kann (Abbildung 1). Dies kommt den Patienten klinisch zugute, indem die steroidbedingten Langzeitnebenwirkungen vermieden werden.

Um die Behandlungsintervalle zu verlängern und die Nebenwirkungen zu reduzieren, wird Bevacizumab zunehmend auch in größeren Therapieintervallen (21-28 Tage versus 14 Tage) und in geringerer Dosierung ( $5 \mathrm{mg} / \mathrm{kg}$ versus $10 \mathrm{mg} / \mathrm{kg}$ Körpergewicht) eingesetzt. In mehreren retrospektiven Studien zeigte dieses Vorgehen und sogar ein Absetzen der Substanz bei klinisch stabilem Zustand keine Nachteile [27-29]. Auch eine verzögerte Therapieinitiierung zu Gunsten einer anderen Therapie oder einer Studienteilnahme zeigte keinen Nachteil [30, 31]. Auch die konkomitierende oder sequentielle Gabe von Bevacizumab mit einer ReBestrahlung stellt in einigen Schweizer Zentren eine Therapieoption dar [26, 32].

\section{Ü1: Ausblick}

Zunehmend erhalten Vakzinierungsstrategien auf der einen und Immuntherapien mit den sogenannten „Checkpoint-Inhibitoren“ wie Ipilimumab, Nivolumab und Pembrolizumab auf der anderen Seite Eingang in die Onkologie [33]. Dies gilt auch für primäre Hirntumoren. Das Ziel der Tumorvakzinierung ist die Sensibilisierung des körpereigenen Immunsystems gegen tumorspezifische (Neoantigene, die nur im Tumor exprimiert werden) oder tumorassoziierte Antigene (körpereigenen Proteine, die im Tumor überexprimiert werden). Immuncheckpointinhibitoren demgegenüber blockieren immunsuppressive Moleküle auf den Oberflächen der Tumorzellen und der T-Lymphozyten, die vom Tumor genutzt werden, um das Immunsystem 
zu hemmen. Der Rezeptor des epidermalen Wachstumsfaktors (EGFR, epithelial growth factor receptor) enthält bei $25-30 \%$ der primären Glioblastome eine „in frame“ Mutation (EGFRvIII), die durch die Deletion von 267 Aminosäuren in der extrazellulären Domäne des Rezeptors hervorgerufen wird [34]. Dieses Protein kommt nur in Tumorzellen vor und wird als tumorspezifisches Antigen für Vakzinierungsstudien gegen das GB eingesetzt [35]. Erst kürzlich zeigte die EGFRvIII-Vakzinierung (Rindopepimut) beim rezidivierten GB in einer randomisierten Phase II-Studie (ReACT) in Kombination mit Bevacizumab im Vergleich zu einer Monotherapie mit Bevacizumab als erste Immuntherapie beim GB ein verlängertes mOS (12,0 versus 8,8 Monate) [36]. Die erfolgreiche Kombination einer Vakzinierung in Kombination mit Bevacizumab wird durch eine Hemmung der immunsupprimierenden Wirkung von VEGF erklärt [37]. Auch der Einsatz von Bevacizumab in Kombination mit Immuncheckpointinhibitoren wird zunehmend untersucht. Bevacizumab soll durch seine antiödematösen Eigenschaften eine peritumorale Schwellung verhindern, die durch eine stimulierte Immunreaktion durch Immuncheckpointinhibitoren ausgelöst wird und folglich schwere neurologische Symptome hervorrufen kann. Anders als der Einsatz von hohen Dosen an Kortikosteroiden wird diese gewünschte Immunreaktion durch Bevacizumab vermutlich nicht unterbunden. Diese aufregenden Entwicklungen in der Immuntherapie könnten zukünftig den Einsatz von Bevacizumab zur Behandlung des GB unter anderen Vorzeichen wieder aufblühen lassen.

\section{Ü1: Schlussfolgerungen}

Trotz enttäuschender Studienergebnisse bleibt Bevacizumab in der Rezidivbehandlung des GB weiterhin eine mögliche und in der Schweiz erstattungsfähige Therapieoption. Die Erwartungen an diese Therapie und die potentiellen Nebenwirkungen müssen individuell mit dem betroffenen Patienten besprochen werden. Dies gilt für die Dosierung, dem Therapieintervall und den Zeitpunkt der Therapieinitiierung im Krankheitsverlauf. Günstig wirkt sich auf die Gesamtkonstellation der steroidsparende Effekt der Substanz dar, wodurch eine negative Stigmatisierung der Patienten (Cushing-Syndrom, Steroidakne) und teils schwerwiegende Nebenwirkungen (u.a. Steroidmyopathie, Osteoporose, Hyperglykämie, Arzneimittelwechselwirkungen) vermieden werden können. Nach wie vor ist das GB eine unheilbare Erkrankung mit erheblicher Morbidität und Mortalität und stellt für die betreuenden Angehörigen eine erhebliche Belastung dar. 
Literaturverzeichnis

[1] Wen PY, Kesari S. Malignant gliomas in adults. N Engl J Med 2008; 359: 492-507.

[2] Hottinger AF, Khakoo Y. Neurooncology of familial cancer syndromes. J Child Neurol 2009; 24: 1526-1535.

[3] Gramatzki D, Dehler S, Rushing EJ, et al. Glioblastoma in the Canton of Zurich, Switzerland revisited: 2005 to 2009. Cancer 2016; 122: 2206-2215.

[4] Seystahl K, Wick W, Weller M. Therapeutic options in recurrent glioblastoma-An update. Crit Rev Oncol Hematol 2016; 99: 389-408.

[5] Wick W, Platten M, Wick A, et al. Current status and future directions of anti-angiogenic therapy for gliomas. Neuro Oncol 2016; 18: 315-328.

[6] Norden AD, Drappatz J, Wen PY. Novel anti-angiogenic therapies for malignant gliomas. Lancet Neurol 2008; 7: 1152-1160.

[7] Salmaggi A, Eoli M, Frigerio S, et al. Intracavitary VEGF, bFGF, IL-8, IL-12 levels in primary and recurrent malignant glioma. J Neurooncol 2003; 62: 297-303.

[8] Nam DH, Park K, Suh YL, et al. Expression of VEGF and brain specific angiogenesis inhibitor-1 in glioblastoma: Prognostic significance. Oncol Rep 2004; 11: 863-869.

[9] Beal K, Abrey LE, Gutin PH. Antiangiogenic agents in the treatment of recurrent or newly diagnosed glioblastoma: analysis of single-agent and combined modality approaches. Radiation oncology 2011; 6: 2.

[10] Jain RK, Di Tomaso E, Duda DG, et al. Angiogenesis in brain tumours. Nature reviews Neuroscience 2007; 8: 610-622.

[11] VanderVeldt AAM, Lubberink M, Bahce I, et al. Rapid decrease in delivery of chemotherapy to tumors after anti-vegf therapy: Implications for scheduling of antiangiogenic drugs. Cancer Cell 2012; 21: 82-91.

[12] Gorski DH, Beckett MA, Jaskowiak NT, et al. Blockade of the Vascular Endothelial Growth Factor Stress Response Increases the Antitumor Effects of Ionizing Radiation. Cancer Res 1999; 59: 3374-3378.

[13] Gilbert MR, Dignam JJ, Armstrong TSTS, et al. A randomized trial of bevacizumab for newly diagnosed glioblastoma. N Engl J Med 2014; 370: 699-708.

[14] Chinot OL, Wick W, Mason W, et al. Bevacizumab plus radiotherapy-temozolomide for newly diagnosed glioblastoma. N Engl J Med 2014; 370: 709-722.

[15] Stark-Vance V. Bevacizumab and CPT-11 in the treatment of relapsed malignant glioma. World Fed Neuro-Oncology Meet 2005; 7: 91.

[16] Friedman HS, Prados MD, Wen PY, et al. Bevacizumab alone and in combination with irinotecan in recurrent glioblastoma. J Clin Oncol 2009; 27: 4733-4740. 
[17] Wick W, Weller M, Van Den Bent M, et al. Bevacizumab and recurrent malignant gliomas: A European perspective. J Clin Oncol 2010; 28: e188-e189.

[18] Stupp R, Weller M. Questions regarding the optimal use of bevacizumab in glioblastoma: A moving target. Neuro Oncol 2014; 16: 765-767.

[19] KreisI TN, Kim L, Moore K, et al. Phase II trial of single-agent bevacizumab followed by bevacizumab plus irinotecan at tumor progression in recurrent glioblastoma. $J$ Clin Oncol 2009; 27: 740-745.

[20] Chinot OL, Reardon DA. The future of antiangiogenic treatment in glioblastoma. Curr Opin Neurol 2014; 27: 675-82.

[21] Armstrong TS, Wen PY, Gilbert MR, et al. Management of treatment-associated toxicites of anti-angiogenic therapy in patients with brain tumors. Neuro-Oncology 2012; 14: 1203-1214.

[22] Sandmann T, Bourgon R, Garcia J, et al. Patients with proneural glioblastoma may derive overall survival benefit from the addition of bevacizumab to first-line radiotherapy and temozolomide: Retrospective analysis of the AVAglio trial. J Clin Oncol 2015; 33: 2735-2744.

[23] Taphoorn MJB, Henriksson R, Bottomley A, et al. Health-related quality of life in a randomized phase III study of bevacizumab, temozolomide, and radiotherapy in newly diagnosed glioblastoma. J Clin Oncol 2015; 33: 2166-2175.

[24] Wick W, Brandes A, Gorlia T, et al. Lb-05 Phase III Trial Exploring the Combination of Bevacizumab and Lomustine in Patients With First Recurrence of a Glioblastoma: the EORTC 26101 Trial. Neuro Oncol 2015; 17: v1.5-v1.

[25] Taal W, Oosterkamp HM, Walenkamp AME, et al. Single-agent bevacizumab or lomustine versus a combination of bevacizumab plus lomustine in patients with recurrent glioblastoma (BELOB trial): A randomised controlled phase 2 trial. The Lancet Oncology 2014; 15: 943-953.

[26] Hundsberger $T$, Hottinger AF, Roelcke $U$, et al. Patterns of care in recurrent glioblastoma in Switzerland: a multicentre national approach based on diagnostic nodes. J Neurooncol 2016; 126: 175-183.

[27] Hertenstein A, Hielscher T, Menn O, et al. Impact of tapering and discontinuation of bevacizumab in patients with progressive glioblastoma. J Neurooncol 2016; 129: 533539.

[28] Levin VA, Mendelssohn ND, Chan J, et al. Impact of bevacizumab administered dose on overall survival of patients with progressive glioblastoma. $J$ Neurooncol 2015; 122 : 145-150.

[29] Anderson MD, Hamza MA, Hess KR, et al. Implications of bevacizumab discontinuation 
in adults with recurrent glioblastoma. Neuro-Oncology 2014; 16: 823-828.

[30] Hamza MA, Mandel JJ, Conrad CA, et al. Survival outcome of early versus delayed bevacizumab treatment in patients with recurrent glioblastoma. $J$ Neurooncol 2014; 119: 135-140.

[31] Piccioni DE, Selfridge J, Mody RR, et al. Deferred use of bevacizumab for recurrent glioblastoma is not associated with diminished efficacy. Neuro-Oncology 2014; 16: 815822.

[32] Gutin PH, Iwamoto FM, Beal K, et al. Safety and Efficacy of Bevacizumab With Hypofractionated Stereotactic Irradiation for Recurrent Malignant Gliomas. IJROB 2009; 75: 156-163.

[33] Neagu MR, Reardon DA. An Update on the Role of Immunotherapy and Vaccine Strategies for Primary Brain Tumors. Curr Treat Options Oncol 2015; 16: 1-18.

[34] Hegi ME, Rajakannu P, Weller M, et al. Epidermal growth factor receptor signaling. Curr Biol 2001; 11: R292-R295.

[35] Schuster J, Lai RK, Recht LD, et al. A phase II, multicenter trial of rindopepimut (CDX$110)$ in newly diagnosed glioblastoma: The ACT III study. Neuro-Oncology 2015; 17: 854-861.

[36] Reardon DA, Desjardins A, Schuster J, et al. ReACT: Long-term survival from a randomized phase II study of rindopepimut ( CDX-110) plus bevacizumab in relapsed glioblastoma EGFR Mutation Variant III ( EGFRvIII ). SNO Congr 2015; 33: \#2009.

[37] Osada T, Chong G, Tansik R, et al. The effect of anti-VEGF therapy on immature myeloid cell and dendritic cells in cancer patients. Cancer Immunol Immunother 2008; 57: $1115-1124$. 
Abbildung 1:

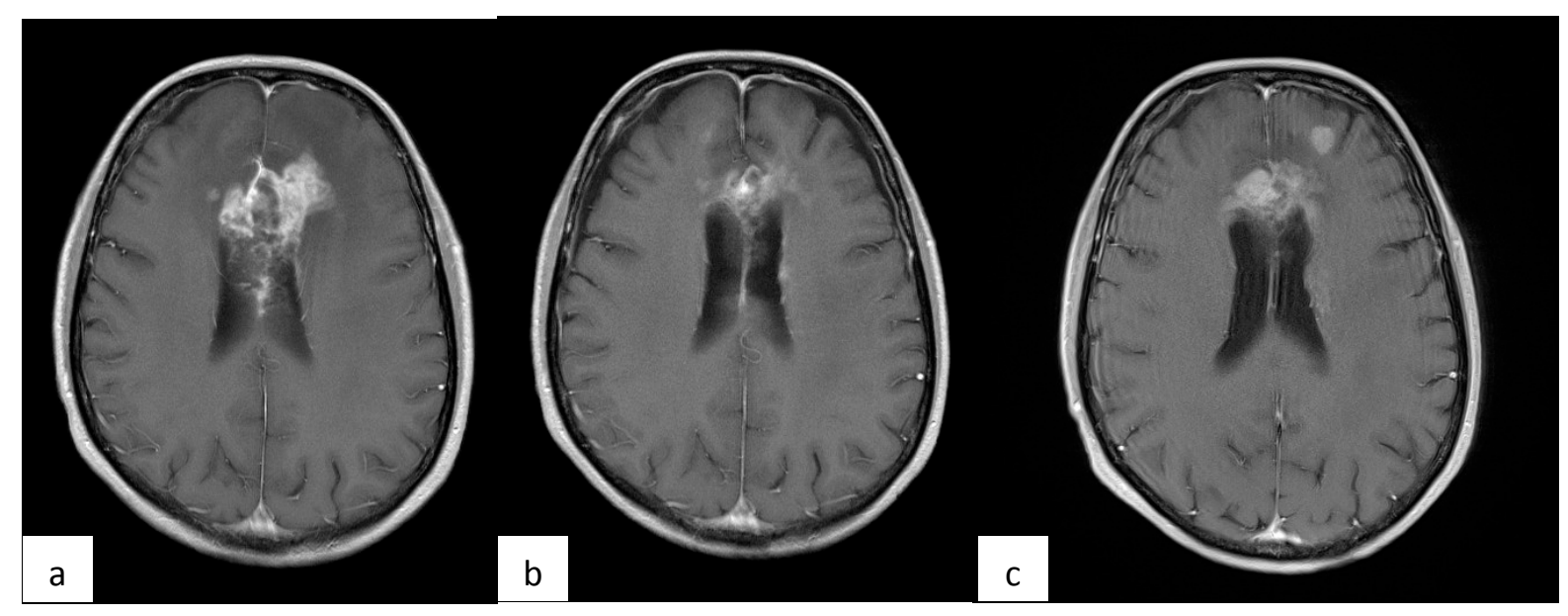

T1 gewichtete, transversales MRT des Neurokraniums mit Gadolinium (Gd) in einer Patientin mit einem rezidivierendem Glioblastom. a) Progressive Gd Aufnahme im vorderen Balken vor Bevacizumab; b) partielle Remission unter Bevacizumab in Kombination mit Lomustin nach 3 monatiger Therapie; c) sekundäre Progression mit multifokaler Gd-Aufnahme nach 6 Monaten Therapie mit Bevacizumab und Lomustin. 
Tabelle 1:

Medianes PFS und OS pivotaler Studien in der Primär- und Rezidivbehandlung des Glioblastoms mit Bevacizumab

\begin{tabular}{|c|c|c|c|}
\hline $\begin{array}{c}\text { Autor } \\
\text { (Patientenzahl) }\end{array}$ & Therapiearm & $\begin{array}{c}\text { mPFS } \\
\text { (Monate) }\end{array}$ & $\begin{array}{c}\text { mOS } \\
\text { (Monate) }\end{array}$ \\
\hline \multicolumn{4}{|l|}{ Primärtherapie } \\
\hline \multirow{2}{*}{$\begin{array}{l}\text { Gilbert et al. } 2014 \\
\qquad(\mathrm{n}=637)\end{array}$} & RT/Tem +Tem & 7,3 & 16,1 \\
\hline & RT/Tem+Tem+Bev & 10,7 & 15,7 \\
\hline \multirow{2}{*}{$\begin{array}{l}\text { Chinot et al. } 2014 \\
\qquad(n=921)\end{array}$} & RT/Tem +Tem & 6,2 & 16,7 \\
\hline & RT/Tem+Tem+Bev & $10,6^{*}$ & 16,8 \\
\hline \multicolumn{4}{|l|}{ Rezidivtherapie } \\
\hline \multirow{2}{*}{$\begin{array}{l}\text { Friedmann et al. } \\
2009 \\
\qquad(n=167)\end{array}$} & Bev & 4,2 & 9,2 \\
\hline & Bev+Iri & 5,6 & 8,7 \\
\hline \multirow{2}{*}{$\begin{array}{l}\text { Wick et al. } 2016 \\
\qquad(n=437)\end{array}$} & Lom & 1,5 & 9,1 \\
\hline & Lom + Bev & $4,2^{*}$ & 8,6 \\
\hline
\end{tabular}

*, statistisch signifikant; RT, Radiotherapie; Tem, Temozolomid; Bev, Bevacizumab; Lom, Lomustin; Iri, Irinotecan 